\title{
The Orientation of Extragalactic Radiosources Relative to the Optical Axes of their Host Galaxies
}

\author{
R. Andreasyan ${ }^{1}$ \\ Byurakan Astrophysical Observatory, Byurakan, 378433, Armenia \\ H. Sol \\ DARC, Observatoire de Paris, 92195 Meudon Cedex, France
}

\begin{abstract}
The relative orientation of radio and optical axes of radiogalaxies has been analysed for a sample of about 300 sources. Radio major axes have a tendency to align with optical minor axes of the host galaxies for the more elongated radiosources while they appear correlated with optical major axes for the less elongated radiosources.
\end{abstract}

\section{Samples of radiosources and classification}

Many attempts have been done to analyse the relative orientation of radio and optical images of low-redshift elliptical radio galaxies (Palimaka et al. 1979, Bottistini et al. 1980, Guthrie 1980, Valtonen 1983, Andreasyan 1984, Sansom et al. 1987) However, to our knowledge, no statistical trends were found up to now, except within the alternative classification of radiosources introduced by Andreasyan (1984). Following this approach, radiosources can be of two types, (i) the elongated ones for which $\mathrm{K}$, the ratio of major to minor radio axes, is larger than 2.5, and (ii) the stocky ones, for which $\mathrm{K}$ is smaller than 2.5. Here we compare this alternative classification with the standard Fanaroff-Riley (FR) classification and further analyse the trends previously found by Andreasyan for the relative orientation.

The present sample of about 300 sources comprises radio galaxies with published radio maps, and identified with elliptical galaxies mainly brighter than 17 magnitude. Values of the position angles of the radio and optical images and elongation of the radio structure have been collected from the literature, or determined on the maps. FRII type sources appear often elongated (62 of 81 cases) while FRI type sources are preferentially stocky ( 52 of 73 cases). However, the two classifications do not completely overlap.

${ }^{1}$ DARC, Observatoire de Paris, 92195 Meudon Cedex, France 

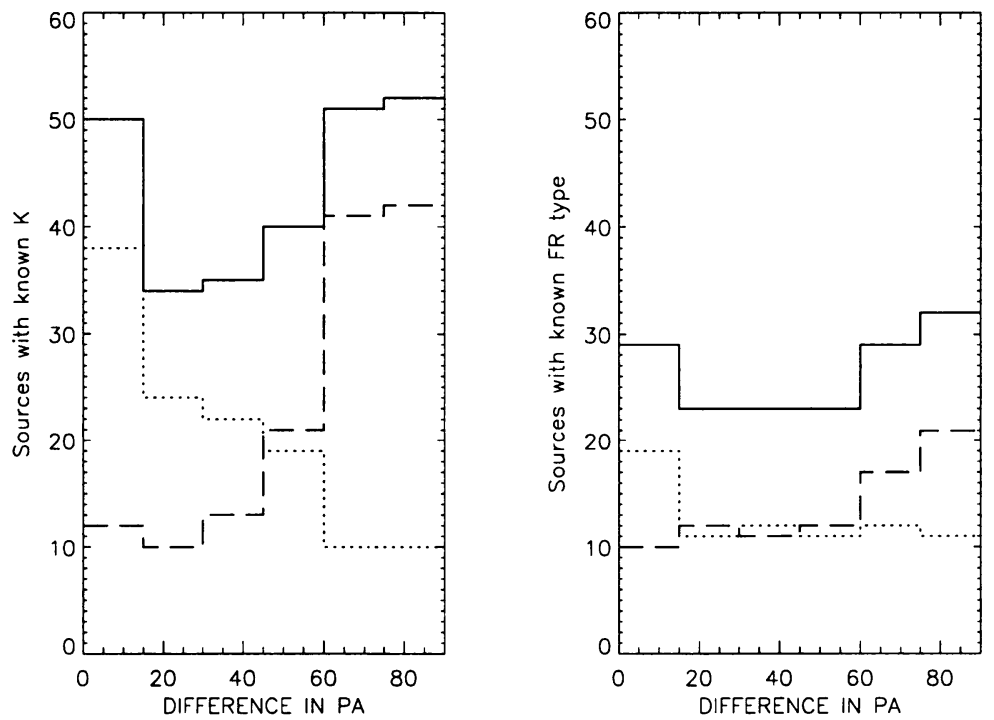

Figure 1. Histogrammes of apparent angle between major radio and optical axes of radiogalaxies, for classification by elongation on the left and for FR classification on the right. Full lines are for whole subsamples, dotted lines for stocky or FRI sources, dashed lines for elongated or FRII sources

\section{Analysis of relative orientation of radio and optical axes}

Histogrammes of the apparent angle between the major radio and optical axes of the radio galaxies are shown in Fig.1, for all the sources and for subsamples of stocky, elongated, FRI and FRII sources. There are two significant peaks, one at zero for the subsample of stocky sources and one at ninety degrees for the subsample of elongated sources. The same two peaks are still visible when using the FR classification, but they are much less significant. So there is a clear tendency to alignment (respectively orthogonality) of major radio axis and optical minor axis for elongated (respectively stocky) radiosources.

The finding of such a correlation between radio and optical axes orientation of radiogalaxies may be important for understanding the mechanism of formation and evolution of these objects.

\section{References}

Andreasyan R.R. 1984, Astrofizika, 21, 409

Bottistini P., et al, 1980, A\&A, 85, 101

Guthrie B.N.G. 1980, Ap\&SS, 70, 211

Palimaka J.J., et al, 1979, ApJ, 231, L7

Sansom A.E., et al, 1987, MNRAS, 229, 15

Valtonen M.J. 1983, Ap\&SS, 90, 207 